\title{
Effects of voids on thermal-mechanical reliability of lead-free solder joints
}

\author{
Lahouari Benabou ${ }^{1, a}$, Van Nhat Le ${ }^{1}$, Zhidan Sun ${ }^{1}$, Philippe Pougnet $^{2}$ and Victor Etgens ${ }^{1}$ \\ ${ }^{1}$ LISV, University of Versailles Saint Quentin-en-Yvelines, 78035 Versailles, France \\ ${ }^{2}$ VALEO, Powertrain Systems, 95892 Cergy Pontoise, France
}

\begin{abstract}
Reliability of electronic packages has become a major issue, particularly in systems used in electrical or hybrid cars where severe operating conditions must be met. Many studies have shown that solder interconnects are critical elements since many failure mechanisms originate from their typical response under thermal cycles. In this study, effects of voids in solder interconnects on the electronic assembly lifetime are estimated based on finite element simulations.
\end{abstract}

\section{Introduction}

Pre-existing voids in solder joints are generated during the manufacturing process and can be classified into various categories. We will focus here on macrovoids which are caused by nonuniform solder shrinkage or by entrapped air due to outgassing during the reflow process. In literature, many conflicting results have been reported regarding the effects of voids on damage in the solder material [1]. The main objective of this study is to investigate separately the effects of void size and location on lifetime of the solder joint. The electronic assembly (Fig. 1a), whose reliability is investigated under thermal cycling, contains lead-free solder interconnections (SAC). Inspection with X-ray tomography (Fig. 1b) reveals that voids are mostly prevalent in the lower solder layer.

\section{Constitutive law and damage model for the solder joint}

The viscoplastic deformation behaviour of the SAC solder is considered using a separated constitutive description where the inelastic strain is decomposed into rate-dependent plastic strain and ratedependent creep strain, $\varepsilon_{\text {in }}=\varepsilon_{\mathrm{p}}+\varepsilon_{\mathrm{c}}$. The elastic perfectly plastic model is a reasonable approximation for the behaviour of $\mathrm{Sn} 3.0 \mathrm{Ag} 0.5 \mathrm{Cu}$ [2]. Contribution of primary creep may be neglected when compared with that of secondary creep [3] and the steady state secondary creep rate can be described by the classical hyperbolic-sine law as $\mathrm{d} \varepsilon_{\mathrm{c}} / \mathrm{d} t=A[\sinh (\xi \sigma)]^{n} \mathrm{e}^{-Q / R T}$.

All solder properties used in computations are provided in Table 1. It should be noted that hardening is only modeled as isotropic. Strain energy density is usually selected as a damage control parameter in

\footnotetext{
${ }^{a}$ Corresponding author: lahouari.benabou@uvsq.fr
}

This is an Open Access article distributed under the terms of the Creative Commons Attribution License 4.0, which permits unrestricted use, distribution, and reproduction in any medium, provided the original work is properly cited. 


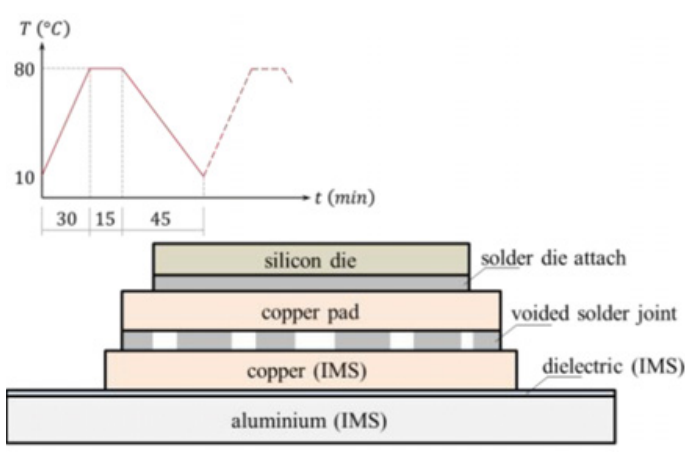

a)

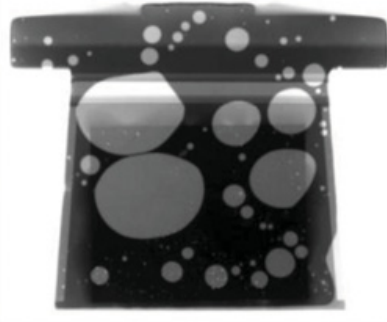

b)

Figure 1. a) Schematic representation of the electronic assembly, b) X-ray tomography inspection of the solder voids.

Table 1. Material parameters of separated model for $\mathrm{Sn} 3.0 \mathrm{Ag} 0.5 \mathrm{Cu}$.

\begin{tabular}{|c|c|c|c|c|c|c|c|}
\hline \multicolumn{4}{|c|}{ Rate-dependent yield stress values } & \multicolumn{4}{|c|}{ Hyperbolic-sine model constants } \\
\hline \multirow{2}{*}{ strain rate $\left(\mathrm{s}^{-1}\right)$} & \multicolumn{3}{|c|}{ yield stress (MPa) at } & \multirow{2}{*}{$A\left(\mathrm{~s}^{-1}\right)$} & \multirow{2}{*}{$\xi\left(\mathrm{MPa}^{-1}\right)$} & \multirow{2}{*}{$n$} & \multirow{2}{*}{$Q / R(\mathrm{~K})$} \\
\hline & $25^{\circ} \mathrm{C}$ & $75^{\circ} \mathrm{C}$ & $125^{\circ} \mathrm{C}$ & & & & \\
\hline $4.02 \mathrm{e}-6$ & 49.0 & 27.0 & 15.0 & $3.1 \mathrm{e} 6$ & $3.7 e-2$ & 7 & $1.0 \mathrm{e} 4$ \\
\hline $4.02 \mathrm{e}-5$ & 55.5 & 37.5 & 23.5 & & & & \\
\hline $4.02 \mathrm{e}-4$ & 59.5 & 42.5 & 26.0 & & & & \\
\hline
\end{tabular}

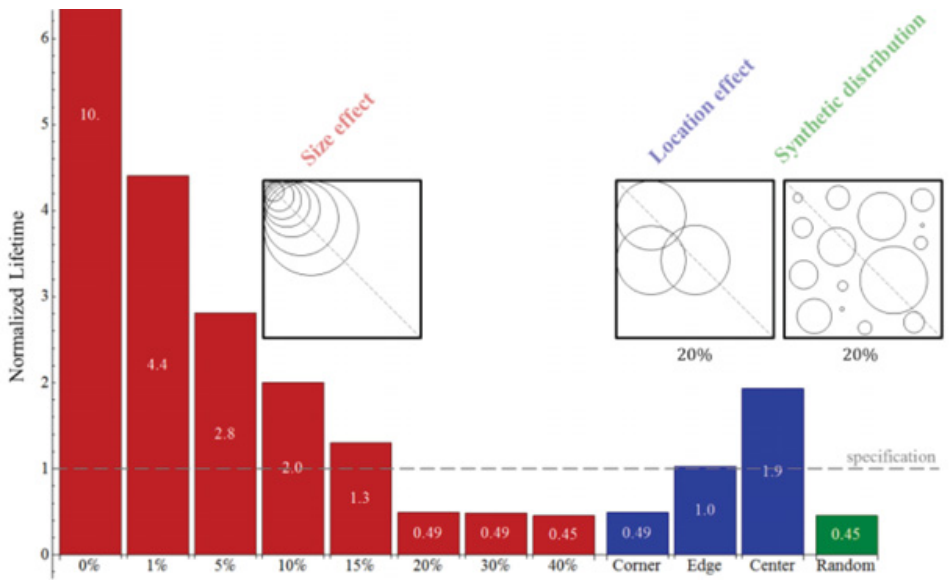

Figure 2. Lifetime normalized with respect to specification of 10000 cycles for different configurations of voids.

fatigue models because it is a comprehensive quantity including effects of both stress and strain. The Morrow's energy-based low cycle fatigue law is given as $N_{\mathrm{f}}^{0.38} \Delta w_{\text {in }}=4.30$.

\section{Finite element simulations}

To reduce sensitivity to meshing, the energy density dissipated per cycle was averaged over a volume of the voided joint including the most critical elements. Figure 2 shows the predicted lifetime for different 
configurations of voided joints. Analyses, carried out with one void at a time, show that the void size is clearly detrimental to reliability. Also, life decreases when a void is located closer to the corner of the joint while its effect is lesser in the middle or near the edge. Finally, lifetime is computed with a synthetic distribution of voids (total volume of $20 \%$ ) based on measured statistical parameters.

\section{References}

[1] L.J. Ladani, A. Dasgupta, J. Electron. Packaging 130, 011008 (2008)

[2] K. Mysore, G. Subbarayan, V. Gupta, R. Zhang, IEEE T. Electron. Pack. 32, 221 (2009)

[3] J.P. Tucker, D.K. Chan, G. Subbarayan, C.A. Handwerker, J. Electron. Mater. 41, 596 (2012) 